\title{
Vintage Effect on the Strain Dependent Dynamics of Ethanol Production in Vineries of Tokaj
}

\author{
Zoltán Kállai1 ${ }^{12 *}$, Gyula Oros ${ }^{3}$ \\ ${ }^{1}$ Research Institute for Viticulture and Oenology, Tarcal, Hungary \\ ${ }^{2}$ Department of Genetic and Applied Microbiology, University of Debrecen, Debrecen, Hungary \\ ${ }^{3}$ Plant Protection Institute HAS, Budapest, Hungary \\ Email: ^kallai.zoltan@tarcalkutato.hu
}

How to cite this paper: Kállai, Z. and Oros, G. (2021) Vintage Effect on the Strain Dependent Dynamics of Ethanol Production in Vineries of Tokaj. Advances in Bioscience and Biotechnology, 12, 31-44. https://doi.org/10.4236/abb.2021.121003

Received: December 8, 2020

Accepted: January 26, 2021

Published: January 29, 2021

Copyright ( 2021 by author(s) and Scientific Research Publishing Inc. This work is licensed under the Creative Commons Attribution International License (CC BY 4.0).

http://creativecommons.org/licenses/by/4.0/

\section{(c) (i) Open Access}

\begin{abstract}
The dynamics of ethanol production of local strains of three yeast species and their ternary mixtures was examined in two Tokaj vineries. Although, the performance of them diverged significantly in first etaps of vinification-up to the utilization of half of the sugar content of grape juice-the variations vintages per vintages surpassed the strain-dependent alterations. The divergence in the latter aspect diminished during the last etap, and the ethanol concentration in young wines fermented by Saccharomyces cerevisiae, $S$. uvarum and Starmerella bacillaris (2 local strains of each) and their mixtures did not vary considerably (c.v. $4.2 \%$ ). The vinification of grape juice performed more rapidly in fermentors inoculated with strains of $S$. cerevisiae, $S$. uvarum and St. bacillaris as well as with their mixtures than in spontaneously initiated ones by wild mycoflora in each vintage. The strains responded in different manners to conditions vintage per vintage, however, their ternary mixtures always fermented more intensively the grape juice than the strains alone. The strains affected the dynamics of alcohol production to different extents, but the alterations between them exceeded the variation between the average effects of the species. The circumstances of vinification significantly influenced the subsequent events of fermentation, but the maximum intensity of ethanol production was inversely proportional to the time required to start alcohol production ( $\mathrm{p}>0.05)$, similar to that observed in the laboratory under strictly controlled micro-vinification experiments. The maximum intensity of ethanol production (MIE) varied between $0.64-2.59 \mathrm{mM}$ ethanol per hour. The coefficients of second-order polynomial equations describing the dynamics of alcohol production in both laboratory micro-scale and medium-scale experiments in cellars revealed similar correlations regarding
\end{abstract}


the interaction of factor groups regulating the process: the constant (timeindependent) and secondary (time-dependent) coefficients of these polynomes counteracted to the primary (time dependent) ones strictly in the strain-dependent manner, and the role of these three factors groups varied also in a strain dependent manner during the vinification process independently of the varying circumstances in three vintages.

\section{Keywords}

Yeast, Vinification, Mixed Fermentation, Ethanol Production, Dynamics, Tokaj

\section{Introduction}

Formerly we revealed groups of factors regulating the ethanol production during fermentation of grape juice in microscale vinification model carried out in highly controlled laboratory conditions [1]. Although the dynamics of ethanol production of wine yeasts examined in these model experiments varied significantly (c.v. 25\%) for Saccharomyces cerevisiae, S. uvarum and Starmerella bacillaris (21, 2 and 2 strains, respectively), the ethanol concentration in young wines fermented did not vary considerably (c.v. 1.9\%). All of them produced significantly higher amounts of ethanol than the type strain [ATCC 26108] of $S$. cerevisiae. The lag phase varied between 33 and 123 hours, while the time requested to produce half of the final ethanol concentration varied between 67 and 294 hours. Moreover, the intensity of maximum ethanol production (MIE) varied between $0.81 \%-4.56 \%$ ethanol per day. Nowadays the property-based application of $S$. uvarum and St. bacillaris strains in wine making technology have been positioned in similar range with $S$. cerevisiae strains [1].

The dynamics of ethanol production could be described by second-order polynomial functions, which have predictive power $(\mathrm{p}<0.001)$ when calculating both Lag phase and End point of fermentation. The constant and secondary coefficients of these functions counteracted to the primary one strictly in strain dependent manner, and the role of these three factor groups also varied in strain-dependent manners during the vinification process. Near linear trend was manifested when interactions of these regulating factor groups were compared, and the position of strains fitted well independently on their origin and taxonomic position.

The main purpose of this work was to test the above findings in semi- industrial conditions as well as to study the effect of environmental conditions changing vintage per vintage.

\section{Materials and Methods}

Data on maintenance, origin and methods of authentication of wine yeast strains used in model experiments (Table 1) and experimental methods were reported 
Table 1. Kinetic parameters of the fermentation dynamics of wine yeast strains.

\begin{tabular}{|c|c|c|c|c|c|c|c|c|c|c|c|}
\hline \multicolumn{2}{|c|}{ Species } & \multicolumn{6}{|c|}{ Ethanol production } & \multicolumn{4}{|c|}{ Sugar utilization } \\
\hline Strains ${ }^{\mathrm{a}}$ & Code $^{\mathrm{b}}$ & $\mathrm{LP}^{\mathrm{c}}$ & $\mathrm{HT}^{\mathrm{c}}$ & $\mathrm{H}-\mathrm{L}^{\mathrm{d}}$ & $\operatorname{SIE}^{\mathrm{e}}$ & $R^{\wedge} 2^{f}$ & $\mathrm{NWE}^{\mathrm{g}}$ & $\mathrm{HT}^{\mathrm{d}}$ & SIS $^{f}$ & $\mathrm{R}^{\wedge} 2^{\mathrm{f}}$ & NWS \\
\hline \multicolumn{12}{|c|}{ Saccharomyces cerevisiae (Desm.) Meyen, 1838} \\
\hline $10-489^{\alpha}$ & $\mathrm{C} 10$ & 54 & 111 & 57 & 1.05 & 0.996 & 11.60 & 104 & 6.20 & 0.995 & 6.70 \\
\hline $10-489^{\alpha}$ & $\mathrm{C} 11$ & 66 & 159 & 93 & 0.64 & 0.999 & 12.56 & 145 & 3.59 & 0.998 & 10.20 \\
\hline $10-489^{\alpha}$ & $\mathrm{C} 12 \mathrm{a}$ & 33 & 67 & 34 & 1.97 & 0.940 & 12.73 & 79 & 12.98 & 0.980 & 2.05 \\
\hline $10-493^{\beta}$ & $\mathrm{C} 12 \mathrm{~b}$ & 53 & 105 & 52 & 1.29 & 0.996 & 12.99 & 103 & 7.88 & 0.996 & 2.75 \\
\hline \multicolumn{12}{|c|}{ Saccharomyces uvarum Beij. 1898} \\
\hline $10-486^{x}$ & $\mathrm{U} 10$ & 49 & 107 & 58 & 1.00 & 0.994 & 11.75 & 102 & 6.21 & 0.993 & 3.10 \\
\hline $10-486^{x}$ & U11 & 55 & 129 & 74 & 0.82 & 0.999 & 12.73 & 132 & 5.11 & 0.997 & 3.50 \\
\hline $10-486^{x}$ & $\mathrm{U} 12 \mathrm{a}$ & 78 & 107 & 29 & 2.59 & 0.949 & 12.24 & 105 & 15.00 & 0.951 & 1.75 \\
\hline $10-499^{\delta}$ & $\mathrm{U} 12 \mathrm{~b}$ & 65 & 97 & 32 & 2.32 & 0.979 & 12.60 & 97 & 13.35 & 0.979 & 1.70 \\
\hline \multicolumn{12}{|c|}{ Starmerella bacillaris (Kroemer \& Krumbholz) F.L. Duarte \& A. Fonseca, 2012} \\
\hline $10-374^{\varepsilon}$ & $\mathrm{Z} 10$ & 102 & 169 & 67 & 0.99 & 0.962 & 11.79 & 157 & 4.24 & 0.950 & 2.20 \\
\hline $10-374^{\varepsilon}$ & $\mathrm{Z} 11$ & 73 & 165 & 92 & 0.68 & 0.999 & 12.80 & 150 & 4.02 & 0.999 & 10.50 \\
\hline $10-374^{\varepsilon}$ & $\mathrm{Z} 12 \mathrm{a}$ & 98 & 144 & 46 & 1.65 & 0.982 & 12.78 & 144 & 9.47 & 0.983 & 2.50 \\
\hline $10-5.11^{\phi}$ & $\mathrm{Z12b}$ & 88 & 132 & 44 & 1.71 & 0.981 & 12.47 & 131 & 9.94 & 0.981 & 1.50 \\
\hline \multicolumn{12}{|c|}{ Mixtures } \\
\hline$\alpha+\chi+\varepsilon$ & M10 & 46 & 86 & 40 & 1.57 & 0.998 & 11.63 & 82 & 9.34 & 0.997 & 2.30 \\
\hline$\alpha+\chi+\varepsilon$ & M11 & 74 & 145 & 71 & 0.92 & 0.994 & 12.88 & 145 & 5.49 & 0.984 & 3.60 \\
\hline$\alpha+\chi+\varepsilon$ & M12a & 42 & 76 & 34 & 2.02 & 0.905 & 12.81 & 89 & 13.83 & 0.971 & 1.40 \\
\hline$\beta+\delta+\phi$ & $\mathrm{M} 12 \mathrm{~b}$ & 54 & 93 & 39 & 1.76 & 0.936 & 12.79 & 93 & 10.23 & 0.939 & 3.00 \\
\hline \multicolumn{12}{|c|}{ Spontaneous } \\
\hline Wild & S10 & 72 & 140 & 68 & 0.92 & 0.950 & 11.83 & 137 & 5.54 & 0.952 & 2.60 \\
\hline Wild & S11 & 81 & 157 & 76 & 0.78 & 0.985 & 11.41 & 160 & 4.49 & 0.971 & 30.50 \\
\hline Wild & S12 & 106 & 153 & 47 & 1.59 & 0.989 & 12.76 & 153 & 9.18 & 0.989 & 1.80 \\
\hline
\end{tabular}

${ }^{a}$ All strains were isolated in Tokaj Wine Region and deposited in the collection of Department of Genetics and Applied Microbiology of University of De-

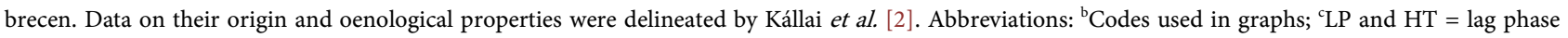
and half time (hours). ${ }^{d}$ Time (hours) requested to produce half of the final ethanol concentration since the end of the lag phase. ${ }^{e} \mathrm{SI}=$ Specific intensity of ethanol production (SIE) and sugar utilization (SIS) at half time (mM/hour). ${ }^{f}$ Determination coefficients of regression curves used for calculation of the parameters (see Figure 1). ${ }^{g}$ Ethanol and sugar concentrations in new wines $(\% \mathrm{v} / \mathrm{v}$ and $\mathrm{g} / \mathrm{L}$, respectively).

in detail by Kállai et al. [2].

\subsection{Semi-Industrial Fermentation in the Winery}

The Furmint grape must was equalized then cleaned with vacuum drum filter. The inoculation concentration was $5 \times 10^{6}$ cells $/ \mathrm{ml}$ of must. The fermentations were carried out in steel tanks filled with $100 \mathrm{~L}$ must in 2010 and 2011. In 2012 the postharvest and the grape must cleaning process was the same, but the fermentation carried out in $50 \mathrm{~L}$ glass carboys. Samples were taken by time course 
given in Figure 1 to observe the dynamics of the fermentation.

\subsection{Analytics}

The alcohol and total sugar concentration was measured with a Bruker Alpha FTIR spectrometer (Bruker Optic GmbH, Germany) and the results were processed with the Bruker OPUS software.

\subsection{Data Analysis}

Fisher's test was applied to evaluate significance of differences between variants at $\mathrm{p}=0.05$ level. The average values of ethanol and sugar concentrations determined in samples taken by time course were used to construct two data matrices
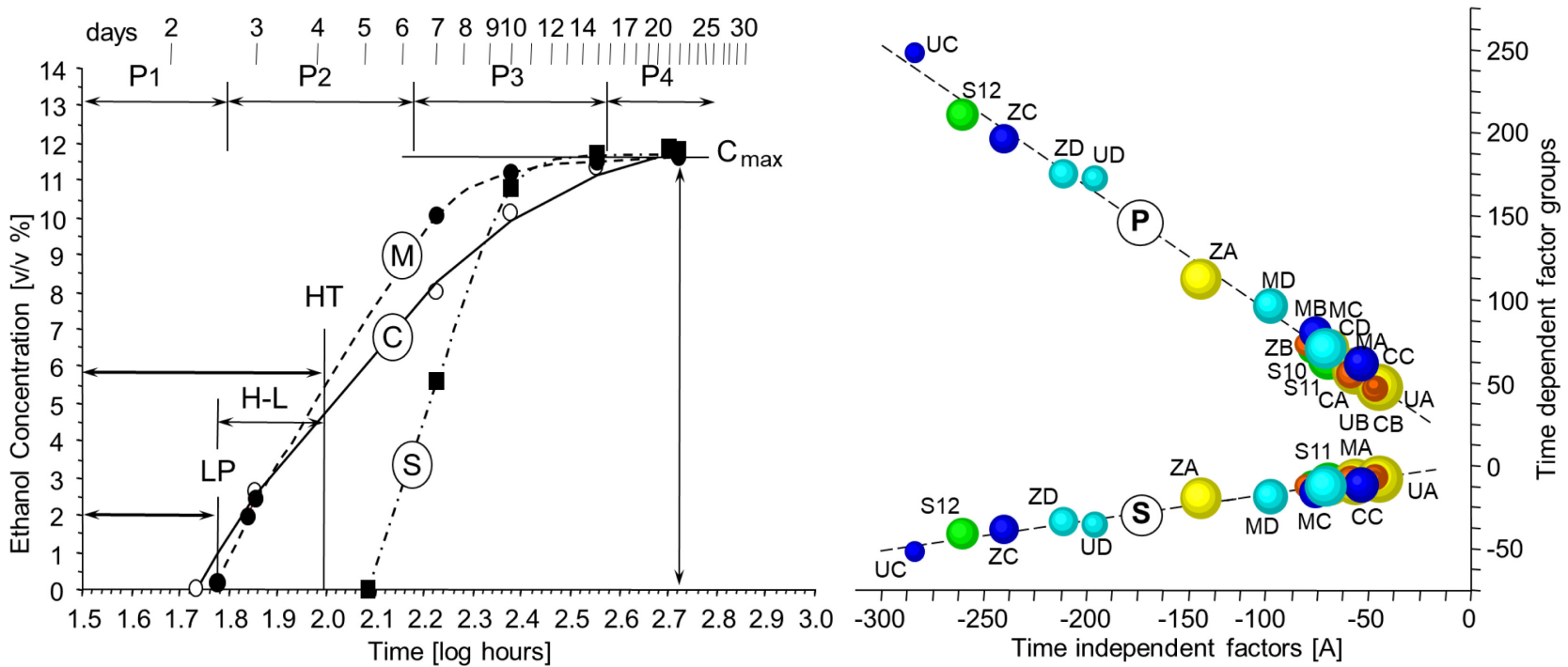

Figure 1. Relationships between factors regulating the strain dependent dynamics of ethanol production. The yeast strains used in experiments were described by Kállai et al. [2]. Left side: Selected samples for demonstration of the process. Dotted lines drawn manually show kinetics of changes of the ethanol production in less and most efficient fermentors, respectively. The process of alcoholic fermentation can be divided into four etaps; $\mathrm{P}_{1}$ - no measurable amount in the medium (lag phase), $\mathrm{P}_{2}$-accelerated growth of concentration, $\mathrm{P}_{3}$-near monotonous growth, $\mathrm{P}_{4}$-retarded growth. $\mathrm{C}_{\max }$ is the ethanol concentration in new wine fermented by mixture of strains, while HT is the half of former value and LP is Lag phase of the same: their values of 2010 vintage were compiled in Table 1 . The arrow $H$ - $L$ marks the interval of the time requested to rich the half of produced ethanol concentration of the end of lag phase. The regressions were calculated of experimentally determined ethanol concentrations (AC) in the medium (measured values at subsequent samplings of $S$. cerevisiae [10-489], terciary mixture of $S$. cerevisiae [10-489], $S$. uvarum [10-486], St. bacillaris [10-374] and wild yeasts (samples are marked with opened and full circles and full quares, respectively): $\mathrm{M}=$ Mixture; $\mathrm{AC}(\%)=-13.731[\text { Time }]^{2}+71.140$ [Time] $-80.410\left(\mathrm{R}^{2}=0.9977\right)$ "Equation $(1)$ "; $\mathrm{C}=$ S. cerevisiae; $\mathrm{AC}(\%)=-10.060$ $\left[_{\text {Time }}^{2}+56.709\right.$ [Time] - $68.081\left(\mathrm{R}^{2}=0.9961\right)$ "Equation $(2)$ "; $\mathrm{S}=$ Spontaneous, $\mathrm{AC}(\%)=-10.865$ [Time] $^{2}+64.084$ [Time] $^{-}$ $81.594\left(\mathrm{R}^{2}=0.9495\right)$ "Equation (3)". where time was given in log hours as measured of the start of fermentation, and determination coefficients $\left(\mathrm{R}^{\wedge} 2\right)$ show the fitting of experimental values to the polynomial functions. Right side: Scatterplot of the coefficients of polynomial equations fitted to experimental values. The regression lines. $\mathrm{P}\left(\mathrm{Pi}=-0.8199 \mathrm{Ci}-1.1659 ; \mathrm{r}^{2}=0.993\right)$ "Equation (4)". S ( $\left.\mathrm{Si}=0.1661 \mathrm{Ci}+1.1942 ; \mathrm{r}^{2}=0.978\right)$ "Equation (5)"; demonstrate relationship between time dependent primary $\left[P\right.$, $\left.\mathrm{b}_{1}\right]$ and secondary $\left[S ; b_{2}\right]$ factors (opened and closed circles, respectively) versus time independent constant factors $[A]$ influencing intensity of actual ethanol production of strains (i). The size of balls is proportional to the time requested for completing the vinification, while the letters $\mathrm{C}, \mathrm{U}, \mathrm{Z}, \mathrm{M}$ and $\mathrm{S}$ mark species cerevisiae, uvarum, zemplinina and their mixtures used for inocolutions as well as spontaneuosly fermented variants (green), while A and B vintages 2010 (lemon) and 2011 (brick). C (ultramarine) and D (azul) mark two sets of the vintage 2012 (see Table 1). 
vintage per vintage, while other characteristics of strains of three species $(S$. cerevisiae, $S$. uvarum and $S$. bacillaris) and their $1+1+1$ mixtures fermenting Furmint juice were put into the separated one.

Data matrices comprising time dependent percentage values were subsequently analyzed by percent of ethanol versus log time regression applying second order polynomial functions to elucidate character of dynamic changes in ethanol production during the fermentation following models described by Sváb [3]. The kinetic parameters (lag phase, half time of alcohol production and end point) were correlated by linear regression. Box plots were used to demonstrate differences between performance of three species ( $S$. cerevisiae, $S$. uvarum and St. bacillaris) and their $1+1+1$ mixtures as well as the wild zymoflora during the vinification process.

Statistical functions of Microsoft Office Excel 2003 (Microsoft, Redmondton, USA) and Statistica 5 program (StatSoft, Tusla, USA) were used for multivariate analysis of data. Graphical presentations of the results of data analysis were edited uniformly in MS Office PowerPoint 2003.

\section{Results and Discussion}

\subsection{The New Wine}

The levels of ethanol (E) and total sugars (TS) could be determined with high accuracy in samples taken during the vinification $\left(\mathrm{F}_{\mathrm{E}}=0.26<\mathrm{F}_{\mathrm{TS}}=1.56<\mathrm{F}_{0.1}=\right.$ 1.71). The alterations in ethanol production between fermentors processed with consortium of wild strains (11.8 - $12.8 \mathrm{v} / \mathrm{v} \%)$ did not surpassed those inoculated with mixed strains $(11.6-12.9 \mathrm{v} / \mathrm{v} \%)$ or with pure cultures $(11.6-13.0 \mathrm{v} / \mathrm{v} \%)$. In general, the effect of vintage $(\mathrm{V})$ on final ethanol concentration was not prominent $\left(\mathrm{F}_{\mathrm{E}, \mathrm{V}}=0.22, \mathrm{p}=0.136\right)$, thus the difference in 2010 and 2012 was only $0.14 \%$ and $0.12 \%$. However, the inoculations in vintage 2011 augmented the ethanol concentrations in new wines at $1.33 \pm 0.14 \mathrm{v} / \mathrm{v} \%$ (Table 1). Similar trend was observed in sugar concentration of new wines, in vintages 2010 and 2011 the differences between wild and inoculated batches were low (max. 0.08 and 0.14 $\mathrm{g} / \mathrm{L}$, respectively), while the residual sugar content in vintage 2011 surpassed the wild one at $23.5 \pm 3.9 \mathrm{~g} / \mathrm{L}$ in inoculated fermentors (Table 1 ). This suggests that the vintage effect manifested itself in the early stages of fermentation by affecting the intensity of sugar consumption $\left(\mathrm{F}_{\mathrm{TS}, \mathrm{V}}=20.2, \mathrm{p}<0.01\right)$, and not only the amount of sugar remaining was increased but also the amount of alcohol produced, as less sugar was used in other processes.

\subsection{Dynamics of Vinification}

The dynamics of ethanol production was demonstrated on selected samples of vintage 2010 (Figure 1). The lag phase of ethanol production in spontaneously fermented grape juice was significantly longer $(72 \mathrm{~h})$ than in fermentors inoculated with either the strain [10-848] or the mixture of three species (54 and 46 hours, respectively), the time requested to surmount toxic concentration to 
yeasts [4] [5] was also longer (8 versus 4 - 5 days). The maximum rate of EtOH production of the pure culture of $S$. cerevisiae $(0.92 \mathrm{mM} / \mathrm{h})$ was lower than the mixtures (the spontaneous fermentation also has been carried out with various cohabiting yeasts). In general, contrary to the near uniform composition of new wines vintage per vintage, the dynamics (D) of ethanol production showed high, strain dependent alterations $\left(\mathrm{F}_{\mathrm{S}}=48.58\right)$ although the character of the process was similar strain by strain $\left(\mathrm{F}_{\mathrm{S}, \mathrm{D}}=1.11\right)$, i.e., after strain dependent length of lag phase started a rapid evolution of ethanol concentration, that followed to over $9 \% \mathrm{v} / \mathrm{v}$ of ethanol in the medium with decreasing intensity up to the ethanol concentration in new wine characteristic to the strain concerned. Although, the vintage effect per se was also prominent $\left(\mathrm{F}_{\mathrm{S}, \mathrm{V}}=6.84\right)$ in this respect (Figure $2(\mathrm{~A})$ ), the dynamics of ethanol production observed in these experiments- carried out in vineries-was similar to that found in highly controlled laboratory conditions [1] [2].

The rate of sugar utilization during lag phase of ethanol production showed high and strain dependent alterations (Figure 3), Nevertheless, the half time of these two processes correlated well (Figure 2(B)). The fermentors separated into two groups by rapidity of the start of ethanol production. The vintage effect in this respect seemingly was insignificant, although the spontaneously fermented batches ranked into group of slow fermenting ones in the first half of vinification (group $\mathrm{U}$ ). The cause of this separation is unclear, as the starting composition of grape juices in subsequent vintages was different $(205,216$ and $222 \mathrm{~g} / \mathrm{L}$ total sugars, respectively), still groups $\mathrm{L}$ and $\mathrm{U}$ have heterogeneous components. One may conclude that the composition of must per se is not necessarily connected
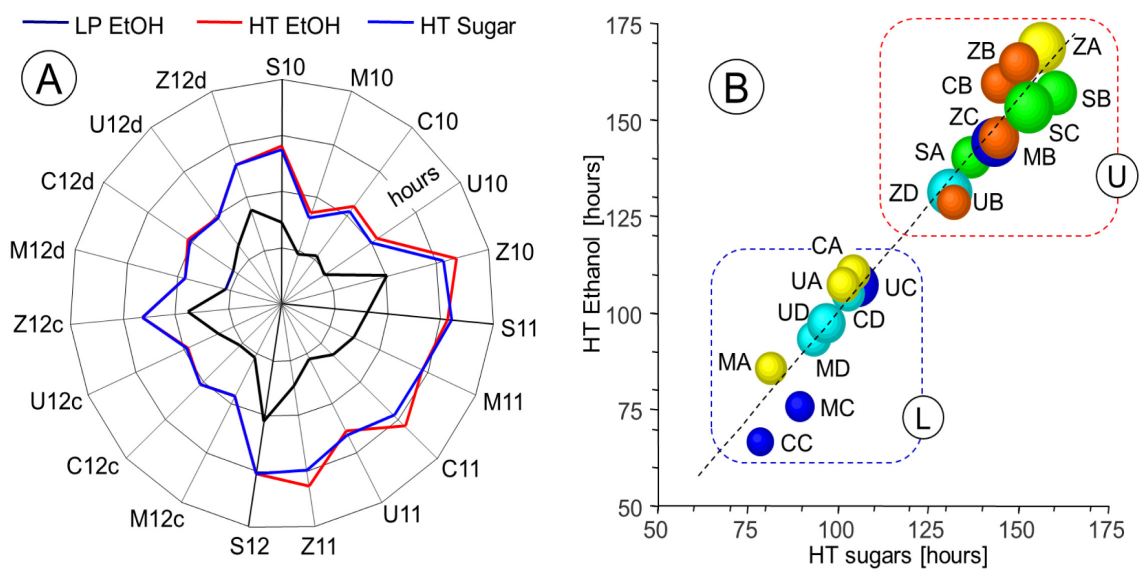

Figure 2. Strain dependent alterations of kinetic parameters of fermentation in various vintages. The codes of strains are given in Table 1. Left side: Subfigure A. Etaps of vinification [units of scale (0-200) are 50 hours]: Lag phase and Half time of ethanol production (black and red lines, respectively), Half time of sugar utilization [blue line]. Right side: Subfigure B. Size of balls is proportinal to lag phase in fermentors (Etap $\mathrm{P}_{1}$ in Figure 1(A)), Vintages 2010 are 2011 marked lemon and brick colors while two variants of 2012 whith ultramarine and azul, respectively. The regression line $\left(\mathrm{y}=1.1151 \mathrm{x}-12.15 ; \mathrm{R}^{2}=\right.$ $0.9563, \mathrm{p}<0.001)$ shows the similarity between dynamics of sugar utilization and ethanol production. 


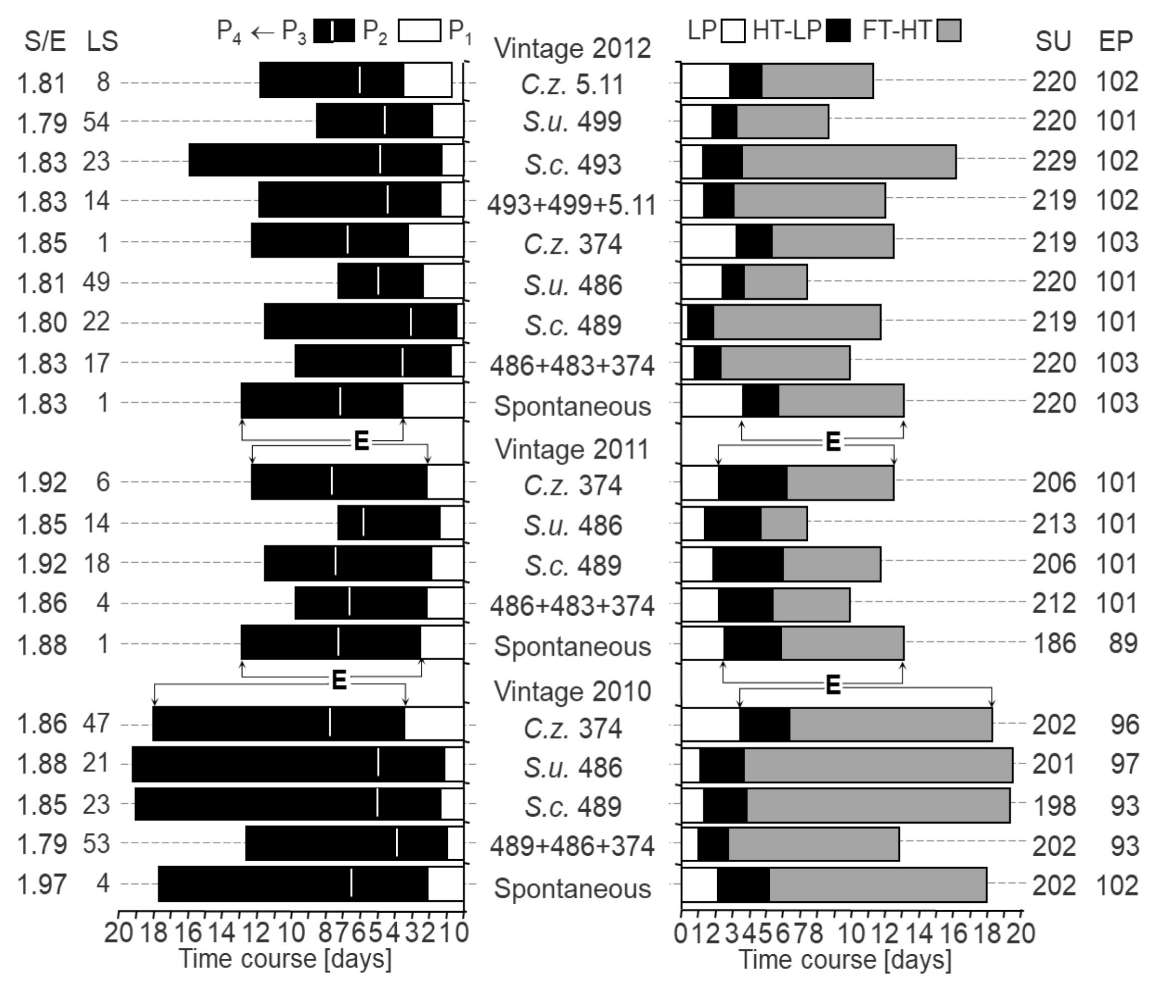

Figure 3. Dynamics of ethanol formation and sugar utilization during vinification of grape must. Left side: Utilization of sugars, Right side: Production of ethanol. Abbreviations: $\mathrm{P}_{1}, \mathrm{P}_{2}, \mathrm{P}_{3}, \mathrm{P}_{4}$ etaps of vinification (see Figure 1); LP = lag phase, HT = half time, HT-LP = time requested to reach the concentration of ethanol of the end of lag phase to half of its final concentration in new wine; FT-HT = time requested to finish the fermentation; S/E = Molar ratio of utilized sugars and produced ethanol; LS = Mass of sugars $[\mathrm{g} / \mathrm{L}]$ utilized in lag phase (SU = total amount of sugar utilized during vinification $[\mathrm{g} / \mathrm{L}]$; $\mathrm{EP}=$ produced mass of ethanol $[\mathrm{g} / \mathrm{L}], \mathrm{E}=$ the period of conditions [hours] with increasing ethanol concentration in the medium, and white lines in black prisms mark the half time of sugar consumption [hours].

to the dynamics of vinification. Examining the process in more details, strain dependent alterations were revealed in the intensity of sugar utilization during lag phase of ethanol production (LS), which can not be related to vintage effect (Figure 3). The time requested to reach the characteristic level of final ethanol concentration showed significant strain dependent differences (c.v. 15\% $\rightarrow 51 \%$ ) that were influenced by vintage effect as well (Figure 3), having been the vinification process more slow in 2010 (11 - 17 days) then either in 2011 or 2012 variants (6 - 10 and 6 - 16 days, respectively). The maximum rates of both sugar utilization (MIS) and ethanol production (MIE) varied within large limits $(3.59 \rightarrow 15$ and $0.64 \rightarrow 2.59 \mathrm{mM} / \mathrm{h}$, respectively) but simultaneously (Figure 4$)$, thus their ratios (MIS/MIE) varied in narrow limits $(1: 1.82 \rightarrow 1: 1.89 \mathrm{mM} / \mathrm{h}$; c.v. $2.57 \%)$.

The vinification process per se run off by strain dependent manner under strong vintage effect (Figure 3 ) that was reflected in differences of maximum rate of ethanol production as well as in the maximum rate of sugar utilization as 

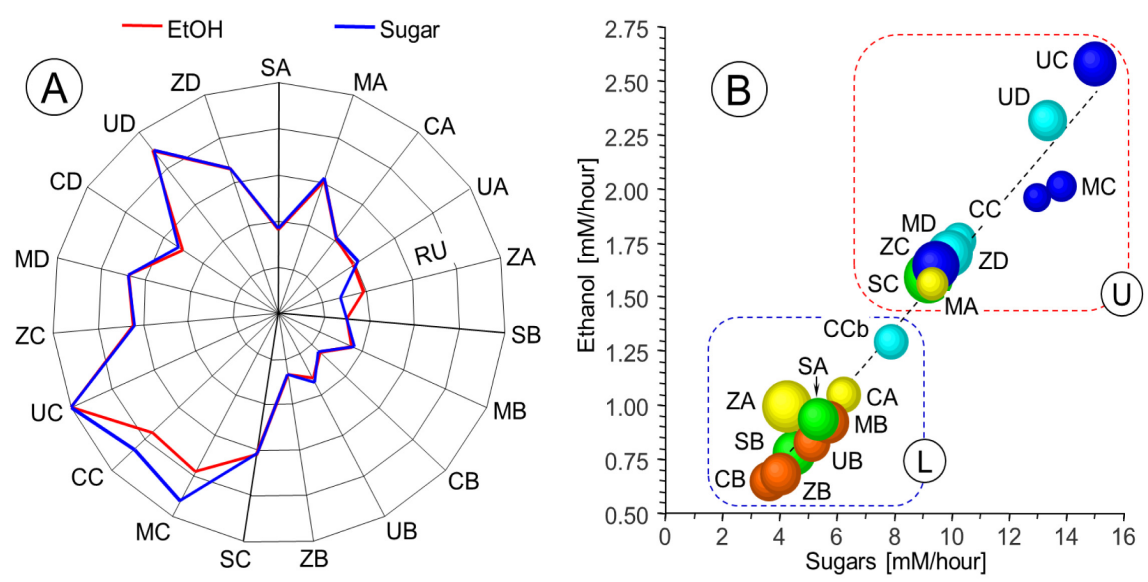

Figure 4. Relationship between ethanol production and sugar utilization of strains. The codes of strains are given in Table 1. Left side: Subfigure A. Maximum capacity of sugar consumption (blue line) and ethanol production (red line), both of these parameters were normalized to their maximum values (S. uvarum [10-486] of vintage 2011!) as related to the set of fermentors (see Table 1 ). Relative units of the scale $(0-1)$ are 0.2 relative unit [RU]. Right side: Subfigure B. Size of balls is proportinal to lag phase in fermentors (Etap $\mathrm{P}_{1}$ in Figure 1(A)), Vintages 2010 are 2011 marked lemon and brick colors while two variants of 2012 whith ultramarine and azul, respectively. The regression $y=0.1563 x+0.0993\left(R^{2}=\right.$ $0.9649, \mathrm{p}<0.001$ ) shows the similarity between sugar consumption and ethanol production by means of maximum capacities of ethanol production versus sugar utilization.

it was demonstrated using normalized values of these two parameters (Figure 4(A)). The great variations caused by vintage effect and differences in species dependent responses were demonstrated on Figure 5. The wild consortium and Stramerella strains proved to be less sensitive to vintage effect than the pure cultures of Saccharomyces strains, and the mixtures of three species. This was reflected both in dynamics of ethanol production (Figure 5(A)) and in the rates of sugar utilization which varied in significantly higher extent than the ethanol production (Figure 5(B)).

\subsection{Role of Hidden Factors}

The dynamics of ethanol production observed in these experiments carried out in vineries was similar to that found in highly controlled laboratory conditions [1] with large set of yeast strains [6]. Studying various mathematical models for description of the dynamics of ethanol production we found the second-order polynomial function being most suitable to predict the result of vinification [2]. This model, proposed by several authors [7] [8] as well, permits to weigh the role of constant, primary (linear), and secondary (quadratic) effects as well as to analyze their relationships in strain-dependent manner (Figure 1 and Table 3). The coefficients of polynomial function describing the dynamics of ethanol formation during vinification process can be conceptualized as vectors, i.e., sums of various factors influencing the ethanol-producing capacity of yeast cells in the vinification process connecting them to both extracellular and intracellular factors regulating the performance of proper strains [1]. Thus, the actual ethanol 

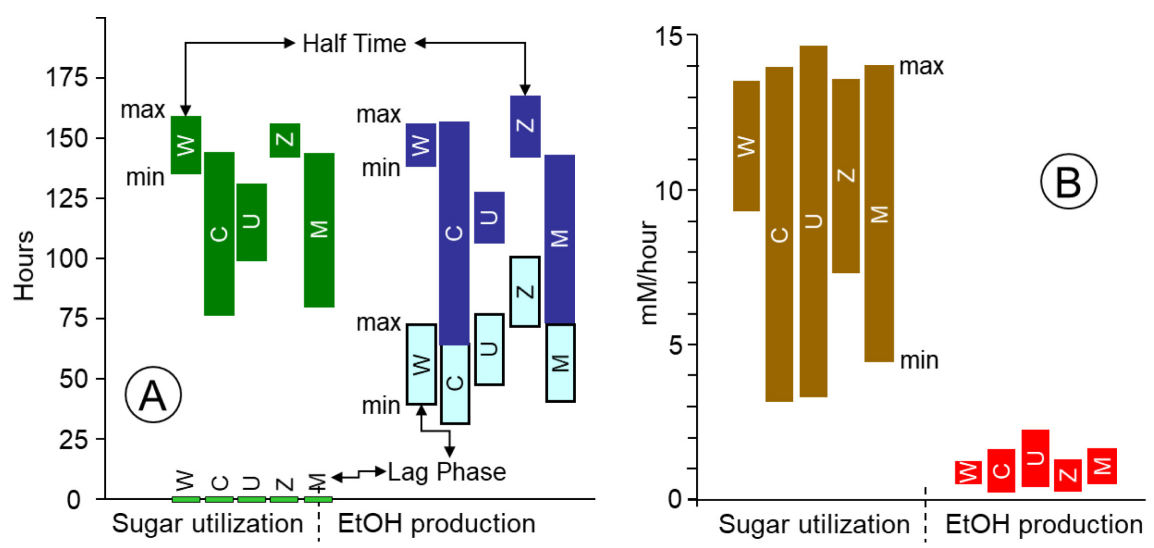

Figure 5. Species dependent variability of the dynamics of sugat utilization and ethanol production. Abbreviations: $\mathrm{W}=$ wild, i.e. spontaneous fermentation, inoculated with $\mathrm{C}=$ S. cerevisiae, $\mathrm{U}=S$. uvarum, $\mathrm{Z}=$ St. bacillaris and $\mathrm{M}=$ mixed inoculation (see Table 1 ). Left side: Subfigure A. Varitations of Lag Phase and Half Time, Right side: Subfigure B. Variations in maximum capacity of sugar utilization and ethanol production.

concentration $(Y)$ is a product of working cell factories and might be extrapolated applying polynomial function $Y_{i}=A+\left[b_{1} \times X_{i}\right]+\left[b_{2} \times X_{i}^{2}\right]$, where $[X]$ is the actual time (i) counted of the start of fermentation, while the $[A]$ is a time-independent constant, which might be related to a group of properties of yeast strains that take part in ethanol production in a time-independent manners as well as not related to responses of cells to the changing environment in the fermentation tank. The influence of both $\left[b_{1}\right]$ and $\left[b_{2}\right]$ manifests in time-dependent mode, and can be considered to be vectors of primary and secondary factor groups, respectively, and these factors most probably take part in the regulation of the responses of cells to changes in environmental conditions. This concept was discussed in details on the example of microvinification experiments [2], where strict trends $(\mathrm{p}<0.01)$ were elucidated in the manifestation of the simultaneous regulatory effects of these groups of factors. The $\left[b_{1}\right]$ group of factors counteracted to both constant $[A]$ and $\left[b_{2}\right]$ groups. Moreover, the influence of factors $\left[b_{1}\right]$ and $\left[b_{2}\right]$ counteracting synchronously in time dependent manner was about two times stronger than the constant ones, meanwhile, the strength of $\left[b_{1}\right]$ group surpasses that of the $\left[b_{2}\right]$ one about five times. The yeast strains fitted precisely to trend lines $(\mathrm{p}<0.01)$ independently on their taxonomic position or other known properties [1]. Similar relationships could be elucidated analyzing results of the experiments carried out in vineries $(V)$ throughout the subsequent vintages where the conditions were not controlled as strictly as in laboratory $(L)$. Plotting time dependent coefficients $\left(b_{1}\right.$ and $\left.b_{2}\right)$ versus time independent one $(A)$ the fermentors fitted strictly to regression line (Figure 1$)$. Comparing the two sets of data, i.e. the coefficients of the polynomial functions obtained of the analysis of results of laboratory and cellar fermentations, a high degree of compliance was demonstrated $\left(t_{V, L}<2\right)$ between the slopes of regression lines (Table 2). However, the intercepts of regression lines are significantly of different size. On our view the above means, that dissimilarities between 
Table 2. Similarities between parameters of polynomial functions describing the dynamics of vinification process carried out in various circumstances.

\begin{tabular}{cccc}
\hline \multirow{2}{*}{ Parameters } & \multicolumn{2}{c}{ Similarities of regression coefficients of polynomial functions ${ }^{\mathrm{a}}$} \\
\cline { 2 - 4 } & PL vs CA & SSQ vs C & SSQ vs PL \\
\hline Intercept (a) of linear funtion $\mathrm{Y}=\mathrm{mX}+\mathrm{a}$ & & \\
Laboratory $(\mathrm{n}=25)$ & $-9.746 \pm 10.944$ & $5.074 \pm 2.013$ & $3.271 \pm 0.149$ \\
Vineries $(\mathrm{n}=19)$ & $-1.169 \pm 3.680$ & $2.152 \pm 1.656$ & $2.037 \pm 0.451$ \\
$\mathrm{t}_{\mathrm{L}, \mathrm{V}}$ & 3.66 & 18.77 & 11.24 \\
$\mathrm{p}$ & $0.01-0.001$ & $<0.001$ & $<0.001$ \\
Slope $(\mathrm{m})$ of linear funtion $\mathrm{Y}=\mathrm{mX}+\mathrm{a}$ & & $-0.2121 \pm 0.0206$ \\
Laboratory $(\mathrm{n}=25)$ & $-0.8585 \pm 0.0294$ & $0.1795 \pm 0.0496$ & $-0.2102 \pm 0.0172$ \\
Vineries $(\mathrm{n}=19)$ & $-0.8445 \pm 0.0207$ & $0.1765 \pm 0.0376$ & -0.33 \\
$\mathrm{t}_{\mathrm{L}, \mathrm{V}}$ & -1.85 & 0.23 & $>0.1$ \\
$\mathrm{p}$ & $0.05-0.1$ & $>0.1$ & \\
\hline
\end{tabular}

${ }^{\mathrm{a}}=$ Coefficients of the polynomial functions describing the dynamics of ethanol productind were imported of Kállai et al. 2019 [2]. The two sets overlapped partially, as 17 strains involved into laboratory experiments were old isolates [1] [2] [9]. $\mathrm{CA}_{1-\mathrm{n}}=$ Constant; $\mathrm{PL}_{1-\mathrm{n}}=$ Primary (linear, $\mathrm{b}_{1}$ ); SSQ $\mathrm{Q}_{1-\mathrm{n}}=$ secondary (squared, $\mathrm{b}_{2}$ ) coefficients of the polynomial functions $\left(\mathrm{Y}=\mathrm{A}+\mathrm{b}_{1} \mathrm{X}+\mathrm{b}_{2} \mathrm{X}^{2}\right.$, where $\mathrm{Y}$ is the actual concentration of substances measured in juice at $\mathrm{X}$ hours after inoculation) describing dynamics of ethanol production in fermentors in laboratory or vineries, respectively.

two sets relate mainly to quantitative (time independent) characteristics of the fermentation, while similarities between sets can be related to qualitative characteristic, which are connected to those strain (inoculant) dependent factors that influence the dynamics of fermentation in time dependent manner. Most probably, the interaction of the latter two groups of factors in regulation of the fermentation was more tolerant to changes in circumstances than those affecting quantitatively the process.

The weight of these suspected factor groups changed during the vinification process (Table 3 ). The constant and secondary factors (CA and SSQ $\left[b_{2}\right]$ ) counteracted primary one (PL $\left[b_{1}\right]$ ) during the whole process, although the power of strain dependent differences only in first half of the fermentation was prominent $\left(\right.$ Chi-sqr exp $>$ Chi-sqr $\left._{0.05}=28.8\right)$. Seemingly, the importance of metabolic processes not related to ethanol production became sidelined, and the significance of strain-dependent differences has been reduced in medium with ethanol concentration over $5-7 \% \mathrm{v} / \mathrm{v}$. One can conclude that; the negative correlation between maximum intensity of ethanol production and linear component of polynomial function (PL, $b_{1}$ ) indicates that some of the strain dependent factors counteract with ethanol production in time dependent manner during mineralization of organic matters in grape juice. The lack of connection between the content of aroma compounds in new wines and the coefficients of polynomial functions describing the ethanol production suggests also that the strain dependent factor groups regulating the dynamics of ethanol production have low 
Table 3. Connection between etaps of fermentation ${ }^{\mathrm{a}}$ and strain dependent factors of polynomial functions describing dynamics of the ethanol production.

\begin{tabular}{|c|c|c|c|c|c|c|}
\hline \multirow{2}{*}{ Variable (D) } & \multicolumn{3}{|c|}{ Importance of factor groups ${ }^{\mathrm{b}}$} & \multicolumn{3}{|c|}{ Parameters of the equations $s^{c}$} \\
\hline & $\mathrm{CA}$ & PL & SSQ & Chi-sqr. & R-sqr. & Power $^{\mathrm{d}}$ \\
\hline \multicolumn{7}{|l|}{ Time course $e^{e}$} \\
\hline Lag phase (hours) ${ }^{\mathrm{f}}$ & 0.691 & -0.628 & 0.575 & 55.3 & 0.9719 & yes \\
\hline HT-LP & -0.526 & 0.586 & -0.628 & 34.5 & 0.8920 & yes \\
\hline Half time (hours) ${ }^{g}$ & 0.142 & -0.064 & 0.003 & 44.8 & 0.9444 & yes \\
\hline FT-LP & -0.119 & 0.192 & -0.247 & 3.6 & 0.2084 & not \\
\hline FT-HT & 0.177 & -0.102 & 0.045 & 1.4 & 0.0865 & not \\
\hline Full time (hours) ${ }^{\mathrm{h}}$ & 0.159 & -0.082 & 0.022 & 6.2 & 0.3311 & slight \\
\hline \multicolumn{7}{|l|}{ Process } \\
\hline $\mathrm{EtOH}(\mathrm{mM} / \mathrm{h})^{\mathrm{i}}$ & 0.638 & -0.699 & 0.742 & 56.8 & 0.9743 & yes \\
\hline Sugar $(\mathrm{mM} / \mathrm{h})^{j}$ & 0.528 & -0.594 & 0.642 & 50.2 & 0.9609 & yes \\
\hline Utilized Sugars ${ }^{\mathrm{k}}$ LP & 0.194 & -0.266 & 0.328 & 8.9 & 0.4381 & slight \\
\hline HT-LP & -0.647 & 0.704 & -0.743 & 23.3 & 0.7778 & yes \\
\hline FT-LP & 0.215 & -0.175 & 0.133 & 8.9 & 0.4374 & slight \\
\hline \multicolumn{7}{|l|}{ New wine ${ }^{1}$} \\
\hline EtOH (\% v/v) & 0.317 & -0.335 & 0.338 & 5.6 & 0.3012 & not \\
\hline Sugar $(g / L)$ & -0.435 & 0.472 & -0.492 & 8.2 & 0.4094 & slight \\
\hline Aromatics $(\mathrm{g} / \mathrm{L})$ & 0.008 & -0.053 & 0.087 & 10.92 & 0.8136 & not \\
\hline Total Acid (g/L) & -0.361 & 0.418 & -0.464 & 14.91 & 0.8992 & zes \\
\hline
\end{tabular}

${ }^{\mathrm{a}}=$ See Table 1 and Figure $1 .{ }^{\mathrm{b}}=$ Coefficients $(\mathrm{C}, \mathrm{PL}, \mathrm{SSQ})$ of the functions $\left(\mathrm{D}_{1-19}=\left\{[\mathrm{CA}]_{1-19}+[\mathrm{PL}]_{1-19}+\right.\right.$ $\left.[S S Q]_{1-19}\right\}$ ), where $\mathrm{D}_{1-19}=$ dependent variable; $\mathrm{CA}_{1-19}=$ Constant; $\mathrm{PL}_{1-19}=$ Primary (linear); $\mathrm{SSQ}_{1-19}=$ secondary (squared) coefficients of the polynomial functions $\left(\mathrm{Y}=\mathrm{A}+\mathrm{b}_{1} \mathrm{X}+\mathrm{b}_{2} \mathrm{X}^{\wedge} 2\right.$, where $\mathrm{Y}$ is the actual concentration of substances measured in juice at $\mathrm{X}$ hours after inoculation) describing dynamics of ethanol production in the 19 fermentors, respectively. ${ }^{\mathrm{c}}=$ parameters of the multiple linear regression function: $\mathrm{D}=$ $f\left(X_{1}, X_{2}, X_{3}\right)$, where $D$ is a dependent variable of the first column, and $X_{1}, X_{2}, X_{3}$ are the coefficients as given above; ${ }^{\mathrm{d}}=$ importance of strain dependent properties influencing the dynamics of ethanol production; $\mathrm{Chi}^{\wedge} 2_{0.05}=7.81, \mathrm{Chi}^{\wedge} 2_{0.1}=6.2, \mathrm{R}^{\wedge} 2_{0.05}=0.7714 ;{ }^{\mathrm{e}}=$ intervals of vinification process (see Figure 1 and Figure 3$) ;{ }^{\mathrm{f}}=$ strain dependent lag phase; ${ }^{\mathrm{g}}=$ strain dependent time requested to reach the $50 \%$ of the final $\mathrm{EtOH}$ concentration produced by proper strains as measured of the start of fermentation; ${ }^{\mathrm{h}}=$ time requested to reach the final $\mathrm{EtOH}$ concentration produced by proper strain; ${ }^{\mathrm{i}}=$ maximum rate of alcohol production; ${ }^{j}=$ maximum rate of sugar utilization; ${ }^{k}=$ portion of utlized sugars (g) during the given ntervals of vinification process; ${ }^{1}=$ composition of new wines fermented (see Table 1 ).

power in determination of bouquet and flavor of new wines.

\subsection{Future Prospects}

The Tokaj rural viticulture has a centuries-old tradition and is one of the most important wine-growing areas in Hungary. The extreme and unpredictable weather associated with climate change in the last decades has significantly affected the date of harvest compared to the usual times and made it difficult to predict it accurately. These anomalies are new challenges that request appropriate developments in vine cultivation and wine making. Moreover, improvements 
are also needed to meet the changing market requirements.

The vinification of grape juice is conducted by yeasts of the genus Saccharomyces, most frequently by $S$. cerevisiae. This species which genome has been sequenced is also a premier research model system because of its genetic tractability, and an extensive array of molecular technologies having been developed for the genetic manipulation of it [9]. In recent decades, it has become accepted to replace or supplement spontaneous fermentation with traditional autochthonous yeasts with commercially available strains with selected traits to correct processing errors and/or improve the quality of the final product [9] [10].

In order to meet increased demand for meet wines with a more complex taste and aroma the research of starter cultures has focused on non-Saccharomyces species as well [10]. Nevertheless, in spite of the availability of several molecular tools, genetically engineered yeast strains are not yet in use in the commercial wine industry. Especially more data requested on their interactions when applied via co- and sequential inoculation, as we have little knowledge of how the different species and their mixture affect the process of fermentation, its dynamics. Therefore, we consider it is necessary to thoroughly analyze the dynamics of fermentation, because knowing this will allow the process to be controlled and thus also facilitate the planned scheduling of grape processing and winemaking. If we know the analytical parameters of our raw material and we know the fermentation ability of the starter culture we want to apply well, we can predict the duration of fermentation as accurately as possible. Furthermore, we can both the optimal time and the mode of intervention to produce the wine with the desired qualitative indicators.

The production of wine from grapes is unique in many respects, and it is one of the world's oldest biotechnological processes. This process is traditionally not conducted under sterile conditions, and the resident winery microbiota contributes to wine quality and style characteristics of local wines. This resident microbiota which seems to be stable as was shown for Tokaj region and in cellar reconstructs for centuries [11] impacts wine quality and regionality. Although, any modified organism has the potential to become an enduring resident of the winery flora, further in-depth studies are needed to find out exactly how much of the desired properties are ingested during fermentation.

The correlations revealed in our comparative study of the coefficients of the equations describing the dynamics of alcohol production may shed light on the role of differences in the traits of strains. The role of these strain dependent differences is seemingly significant after inoculation, but decreases as vinification progresses, so it is important to determine what biochemical processes these three mathematically distinct groups of factors describe. The functions of several yeast genes have already been elucidated [12]. With these results, we can equip the yeasts with advantageous and valuable properties for industrial use.

The molecular diagnostics opened new possibilities to analyze the autochthonous yeast consortia fermeting the grape juice, and newer fast and inexpensive test methods will hopefully allow their widespread use among little growers 
in the near future. Thus, the rapidly evolving methods of "synthetic biology" will also be widely applicable.

Further experiments are needed to develop a mathematical model that better describes the vinification process [13] [14] in parallel with the development of continuous monitoring tools, which allows to predict the dynamics of fermentation more and more accurately, calculated with the effect of more sophisticated winemaking methods, including the different inoculation methods, the interaction of different yeast species and their mixtures, the supply of nutrients, and the regulation of fermentation cycles at different temperatures affecting the whole fermentation, including the time of its duration.

\section{Conclusions}

The strain dependent traits taking part in regulation of the dynamics of ethanol production acted in vineries in similar manner than in microscale experiments carried out in laboratory, thus in this respect the vintage effect was of minor importance.

The vintage effect affected the dynamics of ethanol production quantitatively, and influenced the process up to the end of rapid evolution of ethanol production.

The vintage effect was more prominent in the cases of fermentation initiated with autochthon zymoflora, while the process was more uniform in artificially inoculated fermentors.

The inoculation improved the process; all inoculated fermentors produced more alcohol than the spontaneous fermentations (11.7 vs $11.8-12.2 \mathrm{v} / \mathrm{v} \%$ ).

By means of mathematical model applied the dynamics of ethanol production proceeded in similar manner in all fermentors either initiated with autochthon zymoflora of local cellars or inoculated artificially.

This study provides the step towards the exploitation of the hidden oenological factors regulating the strain dependent changes in dynamics of ethanol production during vinification process.

\section{Acknowledgements}

The authors thank Anita Kovacs-Bordan for expert technical assistance. This research was financed from the grant VP-4-10.2.2.-15 project in the frameworks of the Széchenyi 2020 program.

\section{Conflict of Interest Statement}

The authors declare that the research was conducted in the absence of any commercial or financial relationships that could be construed as a potential conflict of interest.

\section{References}

[1] Kállai, Z., Antunovics, Z. and Oros, G. (2020) Comparative Evaluation of the Dy- 
namics of Alcohol Production of Wine Yeast Strains Isolated in Tokaj Region. Global Journal of Science Frontier Research D: Agriculture \& Veterinary, 20, 1-22. https://doi.org/10.34257/GJSFRDVOL20IS6PG1

[2] Kállai, Z., Pfliegler, W.P., Mitercsák, J., Szendei, G. and Sipiczki, M. (2019) Preservation of Diversity and Oenological Properties of Wine Yeasts during Long-Term Laboratory Maintenance: A Study of Strains of a Century-Old Tokaj Wine Yeast Collection. Lebensmittel- Wissenschaft \& Technologie, 101, 789-798. https://doi.org/10.1016/j.lwt.2018.12.002

[3] Sváb, J. (1981) Biometric Methods in Research. III. Analysis of the Correlation of Quantitative Variables by Regression Analysis, Mezőgazdasági Kiadó, Budapest, 263-425.

[4] Pascual, C., Alonso, A., Garcia, I., Romay, C. and Kotyk, A. (2004) Effect of Ethanol on Glucose Transport, Key Glycolytic Enzymes, and Proton Extrusion in Saccharomyces cerevisiae. Biotechnology and Bioengineering, 32, 374-378.

https://doi.org/10.1002/bit.260320317

[5] Jiménez, J. and Van Unden, H. (1985) Use of Extracellular Acidification for the Rapid Testing of Ethanol Tolerance in Yeasts. Biotechnology and Bioengineering, 27, 1596-1598. https://doi.org/10.1002/bit.260271113

[6] Soós, I. and Ásvány, A. (1950) Morphological and Physiological Investigation of the Hungarian Wine Yeast Collection. Yearbook of the Research Institute for Ampelology, 10, 255-290.

[7] Boudjema, K., Fazouane-Naimi, F. and Hellal, A. (2015) Optimization of the Bioethanol Production on Sweet Cheese Whey by Saccharomyces cerevisiae DIV13Z087C0VS Using Response Surface Methodology (RSM). Romanian Biotechnological Letters, 20, 10814-10825.

[8] Caldeirao, L., Tanaka, C., Ida, E. and Spinosa, W. (2016) Modeling, Kinetic Study of Bio-Ethanol Production from Soy Protein, Concentrate Byproduct. Food Science and Technology, 36, 369-374. https://doi.org/10.1590/1678-457X.0021

[9] Pretorius, I.S. (2000) Tailoring Wine Yeast for the New Millennium: Novel Approaches to the Ancient Art of Winemaking. Yeast, 16, 675-729.

[10] Jolly, N.P., Augustyn, O.P.H. and Pretorius, I.S. (2006) The Role and Use of Non-Saccharomyces Yeasts in Wine Production. South African Journal for Enology and Viticulture, 27, 15-38. https://doi.org/10.21548/27-1-1475

[11] Magyar, D., Kállai, Z., Sipiczki, M., Dobolyi, C., Sebők, F., Beregszászi, T., Bihari, Z., Kredics, L. and Oros, G. (2017) Survey of Viable Airborne Fungi in Wine Cellars of Tokaj, Hungary. Aerobiologia, 34, 1-15. https://doi.org/10.1007/s10453-017-9505-3

[12] La, A., Du, H., Taidi, B. and Perré, P. (2020) A Predictive Dynamic Yeast Model Based on Component, Energy, and Electron Carrier Balances. Biotechnology and Bioengineering, 117, 2728-2740.

[13] Nosrati-Ghods, N., Harrison, S.T.L., Isafiade, A. and Tai, S.L. (2020) Mathematical Modelling of Bioethanol Fermentation from Glucose, Xylose or their Combination-A Review. ChemBioEng, 7, 68-88.

[14] Miller, K.V. and Block, D.E. (2020) A Review of Wine Fermentation Process Modeling. Journal of Food Engineering, 273, Article ID: 109783.

https://doi.org/10.1016/j.jfoodeng.2019.109783 